\title{
INCORPORATION OF A CIRCULAR BOUNDARY CONDITION INTO THE PROGRAM POISSON*
}

Slomo Caspi, Michael Helm, and L. J ackson Laslett

\author{
Accelerator and Fusion Research Group \\ Lawrence Berkeley Laboratory \\ University of California \\ Berkeley, California
}

March 2, 1984

\footnotetext{
"This work was supported by the Director, Office of Energy Research, Office of High Energy and Nuclear Physics, High Energy Physics Division, U. S. Dept. of Energy, under Contract No. DE-ACO3-76SF00098.
} 
INCORPORATION OF A CIRCULAR BOUNDARY CONDITION INTO THE PROGRAM POISSON*

S. Caspi, M. Helm, L.J. Laslett

Lawrence Berkeley Laboratory University of California Berkeley, California 94720

$\underline{\text { Introduction }}$

Two-dimensional problems in electrostatics or magnetostatics frequently are solved numerically by means of relaxation techniques -- employing, for example, the Programs TRIM or POISSON (see, for example, J. Colonias, "Particle Accelerator Design: Computer Programs," Academic Press, New York, 1974, or the original paper A.M. Winslow, "Numerical Solution of the Quasilinear Poisson Equation in a Non-Uniform Triangular Mesh," J. Comput. Phys. 1, 149-172 (1966)). In many such problems the "sources" (charges or currents, and regions of permeable material) lie exclusively within a finite closed boundary curve and the relaxation process in principle then could be confined to the region interior to such a boundary -- provided a suitable boundary condition is imposed onto the solution at that boundary. The present notes discuss and illustrate the use of a boundary condition of such a nature as to imply the absence of external sources, in order thereby to avoid the inaccuracies and more extensive meshes present when alternatively a simple Dirichlet or Neumann boundary condition is specified on a somewhat more remote outer boundary. The boundary condition to be discussed was proposed by one of us, and some illustrative material presented in collaboration with

*This work was supported by the Director, Office of Energy Research, Office of High Energy and Nuclear Physics, High Energy Physics Division, U. S. Dept. of Energy, under Contract No. DE-AC03-76SF00098. 
V.0. Brady, in early unpublished Lawrence Berkeley Reports (see L.' Jackson Laslett, ESCAR-28, "On a Boundary Condition Applicable to Magnetostatic Relaxation Computations," (November 5, 1975) and Victor Brady and L. Jackson Laslett, LBID-172, "Incorporation of a Circular Boundary Condition for a Magnet with Quadrant Symmetry into the Progran TRIM," (January, 1980)).

The proposed boundary condition $c$ an be introduced in a general manner by reference to one form of Green's theorem for harmonic functions (see ESCAR-28), but may be more simply illustrated by specific use of plane-polar coordinates. Thus, with a circular boundary so located that no external sources are present, the potential function external to that boundary is expressible in the form

$$
c_{0}+\sum_{m=1} r^{-m}\left(c_{m} \cos m \theta+s_{m} \sin m \theta\right) .
$$

in which no positive powers of $r$ occur, and such a relation will permit one to extend the potential to a surrounding concentric circle of somewhat larger radius. If, in practice, values of potential are known at only a finite number of points in the inner circle, then of course only a finite number of harmonic coefficients $\left(C_{m}, S_{m}\right)$ could be evaluated for such trigonometric representation of the potential function -- such a trigonometric series may, however, be adopted to provide adequate estimates of the corresponding values of potential at various points on a near-by surrounding "outer-boundary curve" (Fig. 1).

In performing a relaxation computation on a mesh bounded by such a pair of curves (external to all "sources"), any full relaxation pass through the mesh may be followed by a step wherein the values of potential at points on 
the outer boundary are revised (up-dated) on the basis of a harmonic description of the potential function on the inner curve. Such revised values would then be employed, as boundary values, in proceeding with the next relaxation pass through the mesh. [An analogous procedure of course would be followed if one were to adopt an elliptical coordinate system $(u, v)$, for which harmonic terms would be of the form $e^{-m u}$ times circular functions of argument mv (see ESCAR-28).]

In the work summarized in the present note, we have made a practical application of the techniques just described, with particular application to the use of the relaxation program POISSON as applied to the design of superconducting magnets for advanced particle accelerators. It is evident that in such work one takes advantage of such intrinsic symmetries as may be present in the geometrical configuration and current distribution for the problem of interest. [Thus, in some cases, only terms involving the cosine of arguments $(2 k-1)_{\theta}$ may be required for harmonic development.] One realizes also that, in practice, there may be a large number of mesh points along the inner (circular) curve whereon one construct a harmonic representation of the potential and (especially for circular boundaries) such points may have a quite unequal spacing. Under such circumstances it may well be expedient, as we indicate, to base the analysis on a restricted number of trigonometric coefficients and to compute these coefficients by a weighted least-squares evaluation of the data.

The following note includes a description of the equations introduced into our operating POISSON program, and this material is followed by some illustrative examples that include a test run for which results could be compared with exact results obtained directly from Ampere's law. Additional material includes some early working notes, * that have been attached for 
convenient reference. It has seemed desirable that we summarize this work informally at this time in order that work now in progress (here and elsewhere) relating to magnet design may benefit from consideration of the concepts and techniques illustrated by this note.

The analysis part, following the introduction, deals with two methods, one is a direct approach and the other is a least squares approach. Part two is a Test Example that compares analytical and computed results. Finally in Part three we explain how to use the "Poisson" group on the HP 1000.

Analysis

Consider the case where a circular arc of radius $r=\mathrm{R}-\mathrm{H}$ divides space into two regions (Fig. 1), an inner one which includes all current sources and magnetic iron, and an outer one which is in free space (hereafter referred to as the "universe"). Since the free space region is infinite we shall arbitrarily limit it by a secondary circular arc of radius $r=R$. Both circular

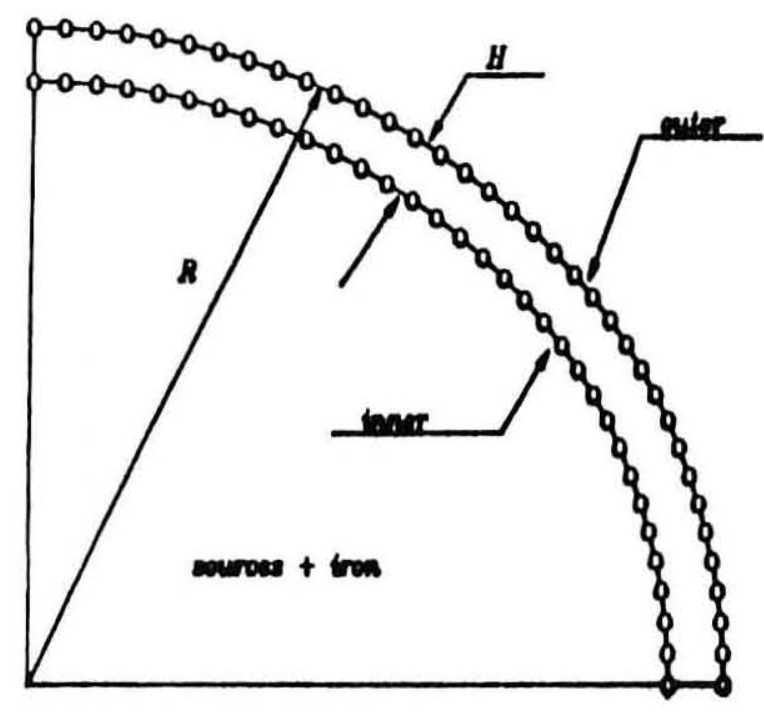

Fig. 1 arcs are an assembly of connecting mesh points such as the one generated by the program LATTICE. If we know the vector potential for each mesh point on $r=R-H$ (e.g. calculated by

*In some of the early work, factors such as $\left(\frac{a}{a-h}\right)^{-n}$ have been replaced by some equivalent small-h approximation. 
POISSON), we would like to find out the vector potential at each mesh point on $r=R$, so that such values may be employed as provisional boundary values in a subsequent relaxation pass through the entire mesh. This is expressed as:

$$
A_{k}^{\text {outer }}=\sum_{n=1}^{N} E_{k n} A_{n}^{\text {inner }}
$$

$A$ is the vector potential and $E$ is a working matrix, and the summation is over the entire mesh points of the inner arc.

In the free space region the vector potential for the symmetries considered here can be expressed as a sum of harmonic terins, each employing powers of $1 / r$.

$$
\begin{gathered}
A_{i}=\sum_{k=1}^{m} c_{k} r^{-\alpha_{k}} \cos \left(\alpha_{k} \theta_{i}\right)=\sum_{k=1}^{m} F_{i k} \cdot c_{k} r^{-\alpha_{k}} \\
F_{i k}=\cos \left(a_{k} \theta_{i}\right)
\end{gathered}
$$

The vector potential $A$ of mesh point $i$ on the circular arc $r$ is defined according to the problem symmetry $\alpha_{k}$ (the equal sign in Eq. (2) is left for the infinite series). (See table on page 11.)

In order to find the $C_{k}$ 's in Eq. (2), a direct approach and a least squares approach are both examined. The direct approach fixes the total number of harmonics employed, $m$, to be the same as the number of node points $\mathrm{N}$, which result in $\mathrm{N}$ equations with $\mathrm{N}$ unknowns. Therefore in a problem where the number of mesh points is large, the number of harmonic terms will be as large. Employing a large number of high harmonics of a vanishing magnitude using a digital computer may result in erro if the mesh points are 
not equally spaced (as will be discussed later). To overturn this limitation the second approach has been developed which is more general and suitable for problems where the number of mesh points is larger than the number of harmonics $(N \gg m)$ and the points are not necessary regularly spaced.

Direct Approach $(\mathrm{N}=\mathrm{m})$

The total number of harmonic terms, $m$, employed is the same as the number of mesh points $N$ on the inner circular arc $r=R-H$. From Eq. (2) solving for $C_{k}$ we get

$$
C_{k}=\sum_{i=1}^{m} r^{a_{k}}\left(F^{-1}\right)_{k i} A_{i}
$$

On the inner circular arc $r=R-H$ we have

$$
c_{k}=\sum_{i=1}^{m}(R-H)^{a_{k}} \cdot\left(F^{-1}\right)_{k i} A_{i}^{\text {inner }}
$$

Substituting Eq. (4) into expression (2) for the outer circular arc $r=R$ we get

$$
A_{j}^{\text {outer }}=\sum_{i=1}^{m} \sum_{k=1}^{m}\left(\frac{R-H}{R}\right)^{a_{k}} F_{j k}\left(F^{-1}\right)_{k i} A_{i}^{\text {inner }}
$$

Expression (5) is rewritten with the following working matrix.

$$
E_{j i}=\sum_{k=1}^{m}\left(\frac{R-H}{R}\right)^{\alpha_{k}} \cos \left(\alpha_{k} \theta_{j}\right)\left(F^{-1}\right)_{k i}
$$


so finally we arrive at

$$
A_{j}^{\text {outer }}=\sum_{i=1}^{\text {In }} E_{j i} A_{i}^{\text {inner }}
$$

Relation (6) sets the vector potential of point $j$ on the outer arc $r=R$ as a linear combination of the potential on the inner arc $r=R-H$ through the working matrix E. For the case where the mesh points on the inner arc are "regularly" spaced, in the sense shown in Fig. 2 the inversion of $F$ is direct and the working matrix reduces to:

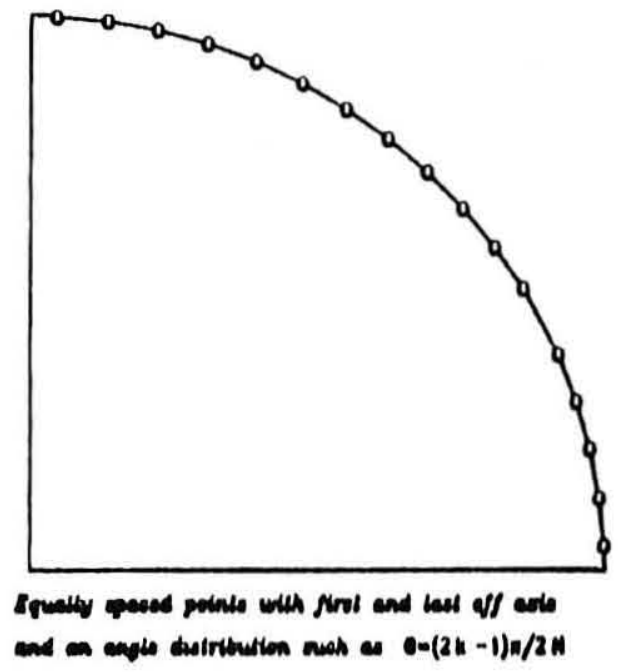

Fig. 2

$$
E_{j i}=\frac{2}{N} \sum_{k=1}^{m}\left(\frac{R-H}{R}\right)^{\alpha_{k}} \cos \left(\alpha_{k} \theta_{j}\right) \cdot \cos \left(\alpha_{k} \theta_{i}\right)
$$

Least Squares Approach $(\mathrm{N} \gg \mathrm{m})$

This method employs a list squares method to calculate the $C_{k}$ 's. From Eq. (2)

$$
\operatorname{minimize:} \quad \frac{1}{2} \sum_{i=1}^{N} w_{i}\left[\sum_{k=1}^{m} c_{k} r^{-\alpha_{k}} \cos \left(a_{k} \theta_{i}\right)-A_{i}\right]^{2}
$$

or 


$$
\frac{1}{2} \sum_{i=1}^{N} W_{i}\left[\sum_{k=1}^{m} F_{i k} c_{k} r^{-\alpha_{k}}-A_{i}\right]^{2} ; \quad F_{i k}=\cos \left(\alpha_{k} \theta_{i}\right)
$$

$W_{j}=$ weight associated with each point (e.g. the relative ratio of the points spacing).

Following the minimization process with respect to the $C_{k}$ we get:

$$
\sum_{j=1}^{m} M_{i j} c_{j} r^{-\alpha}=v_{i}
$$

where

$$
\begin{aligned}
& M_{i j}=\sum_{n=1}^{N} w_{n} \cos \left(\alpha_{i} \theta_{n}\right) \quad \cos \left(\alpha_{j} \theta_{n}\right) \\
& \left.v_{i}=\sum_{n=1}^{N} w_{n} \cos \left(a_{i} \theta_{n}\right) \quad A_{n}\right\} \quad i, j=1,2, \ldots, m
\end{aligned}
$$

Solving for $C_{j}$ on the inner arc $r=R-H$ we get

$$
C_{j}=\sum_{i=1}^{m}(R-H)^{\alpha}\left(M^{-1}\right)_{j i} v_{i}^{i n n e r}
$$

Using Eq. (2) on the outer arc $r=R$

$$
A_{k}^{\text {outer }}=\sum_{j=1}^{m} F_{k j} C_{j} R^{-a_{j}}
$$

and substituting the relations for $F_{k j}, C_{j}$ and $v_{i}^{\text {inner }}$ we arrive at 


$$
A_{k}^{\text {outer }}=\sum_{j=1}^{m}\left(\frac{R-H}{R}\right)^{\alpha_{j}} \cos \left(\alpha_{j} \theta_{k}\right) \sum_{i=1}^{m}\left(M^{-1}\right)_{j i} \sum_{n=1}^{N} W_{n} \cos \left(\alpha_{i} \theta_{n}\right) A_{n}^{\text {inner }}
$$

Employing the working matrix $E_{k n}$, relation $(10)$ is rewritten as:

$$
A_{k}^{\text {outer }}=\sum_{n=1}^{N} E_{k n} A_{n}^{\text {inner }}
$$

where

$$
E_{k n}=\sum_{i=1}^{m} \sum_{j=1}^{m}\left(\frac{R-H}{R}\right)^{\alpha_{j}} w_{n}\left(M^{-1}\right)_{j i} \cos \left(\alpha_{j} \theta_{k}\right) \cos \left(\alpha_{i} \theta_{n}\right)
$$

In the case where the points on the inner arc $r=\mathrm{R}-\mathrm{H}$ are "regularly" spaced (Fig. 2) then $\cos \left(\alpha_{i} \theta_{n}\right)$ are orthogonal with respect to summation over $\theta$, and the $M$ matrix reduces to:

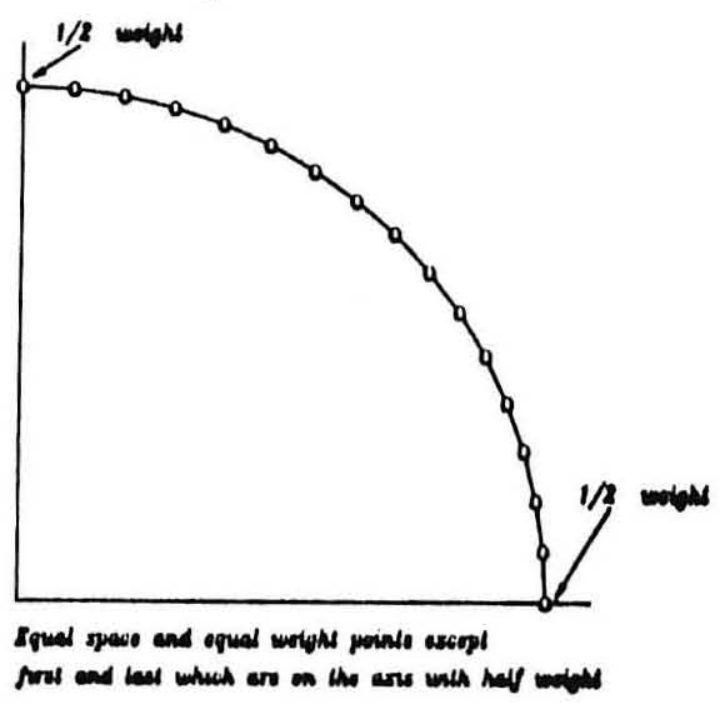

Fig. 3 


$$
\left.\begin{array}{l}
M_{i j}=\left\{\begin{array}{ll}
N \delta_{11} & a \neq 0 \\
\frac{N}{2} \delta_{i j} & a \neq 0
\end{array}\right\} \text { for regularly spaced points } \\
M_{i j}=\left\{\begin{array}{ll}
(N-1) & \delta_{11} \\
(N-1) & \delta_{m, m}
\end{array}\right\} a=0 \\
\frac{N-1}{2} \delta_{i j}
\end{array}\right\} \begin{aligned}
& \text { first and last point are on the axes } \\
& \text { with } 1 / 2 \text { weight; Fig. } 3
\end{aligned}
$$

where $\delta$ is the Kronecker delta.

Substituting the inversion of $M$ into the working matrix, we get

$$
E_{k n}=\frac{2}{N} \sum_{i=1}^{m}\left(\frac{R-H}{R}\right)^{\alpha_{i}} \cos \left(\alpha_{i} \theta_{k}\right) \cos \left(\alpha_{i} \theta_{n}\right)
$$

This expression is identical to the one derived by the direct method for "regularly" spaced points. 
Listed is a table for the $a_{k}$ expression used in several geometries.

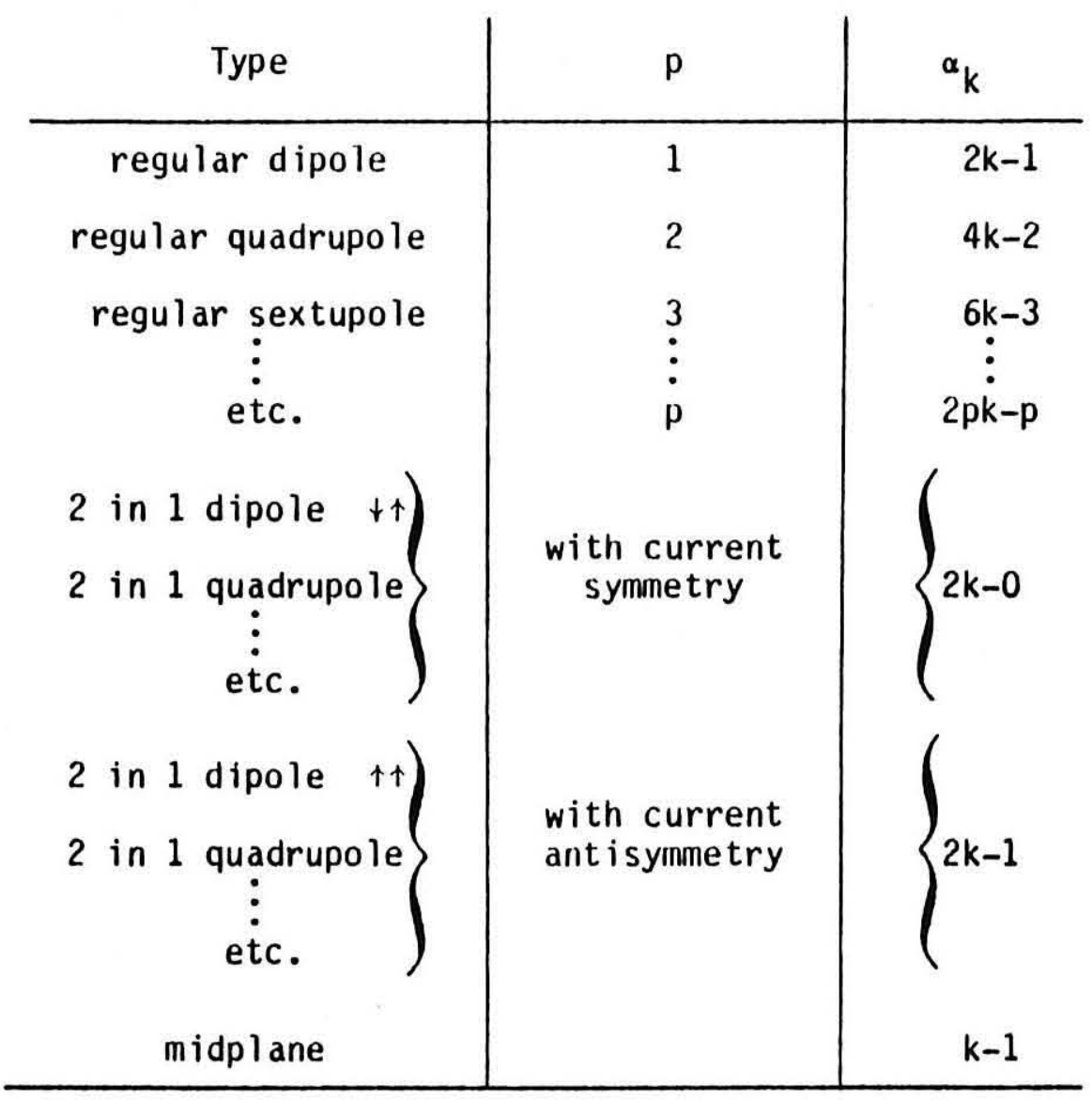




\section{Test Example}

In order to compare the results using our proposed method and the one calculated analytically, we solved a number of simple cases (no iron). In the first example we provide the solution for the vector potential in a 2-in-1 dipole arrangement employing current sheets. In the other test cases we solve a regular single; dipole, quadrupole and sextupole; all employing single current lines per pole.

2-in-1 Dipole Employing Current Sheet

Sketched below is the geometry used, followed by the solution.
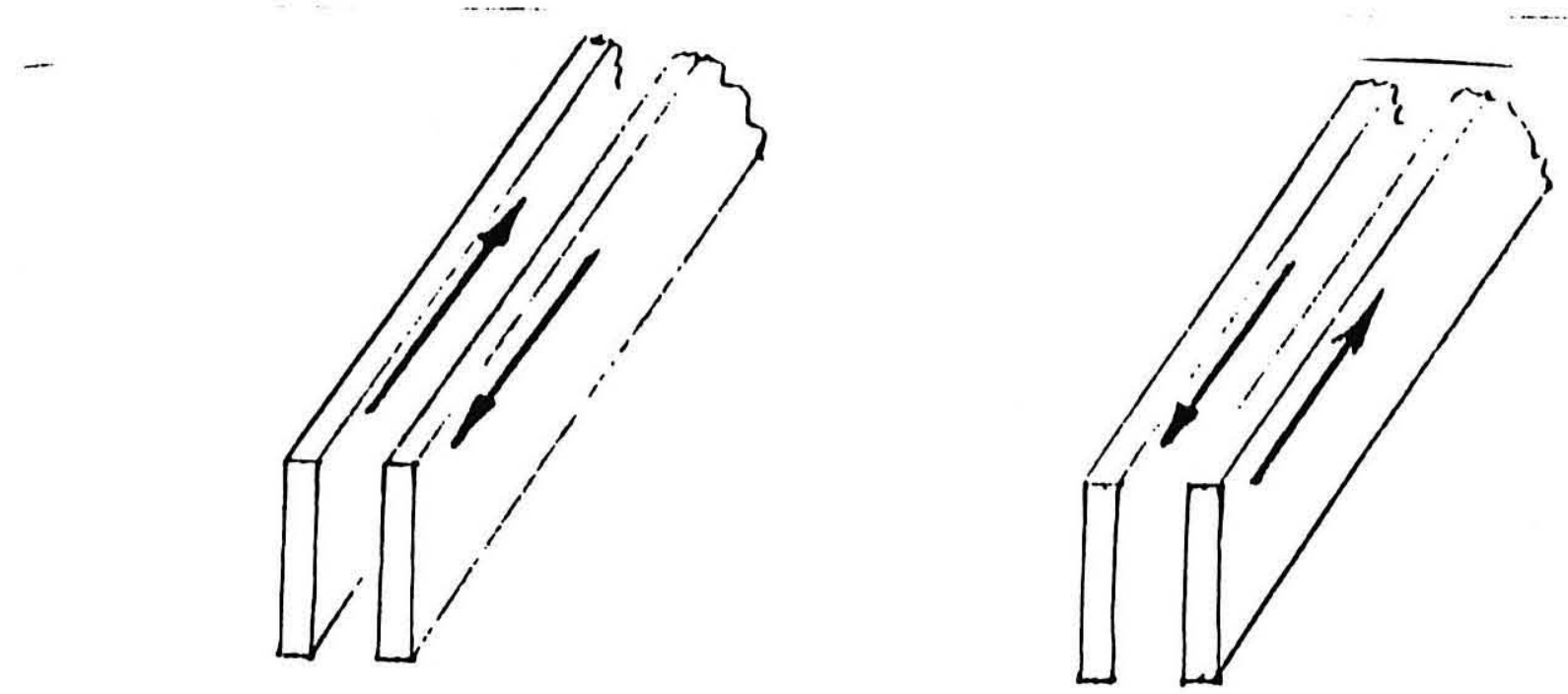

Employing current sheets (infinite length, infinitessimal thickness)
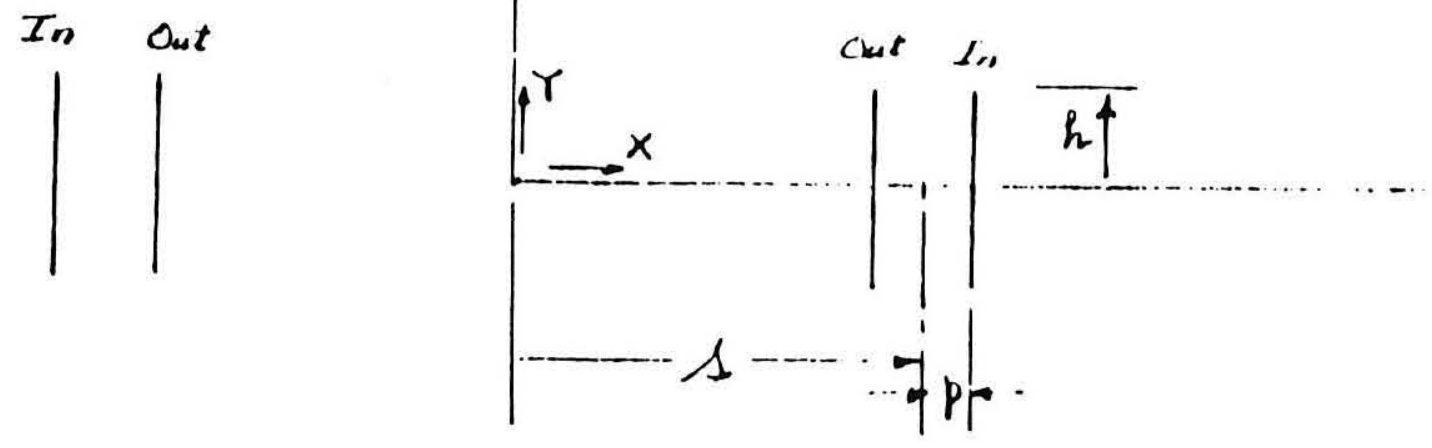


$$
\begin{aligned}
& \text { linear dimensions in } \mathrm{cm} \text {, } \\
& \mathrm{J} \text { in amps/cm } \\
& B \text { in gauss } \\
& A_{Z} \text { in gauss.cm }
\end{aligned}
$$

we employ

$$
\mu_{0}=\frac{4 \pi}{10}
$$

If

$$
\begin{aligned}
& \text { linear dimensions in meter, } \\
& \mathrm{J} \text { in amps/m } \\
& B \quad \text { in Tesla } \\
& A_{2} \text { in Tesla.m }
\end{aligned}
$$

we employ

$$
\mu_{0}=\frac{4 \pi}{10} .
$$

but we shall adopt the fomrer choice.

For

$$
\begin{gathered}
\nabla^{2} \vec{A}=-\mu_{0} j \text { with } \nabla \cdot \vec{A}=0, \\
\vec{A}=\frac{\mu_{0}}{4 \pi} \iiint \frac{j}{R} d v^{\prime} .
\end{gathered}
$$

For a single wire pair (long wires), 


$$
\begin{aligned}
\vec{A} & =\frac{\mu_{0}}{4 \pi} I \hat{e}_{z} \int_{-\infty}^{\omega}\left[\frac{d z}{\sqrt{p_{+}{ }^{2}+z^{2}}}-\frac{d z}{\sqrt{p_{-}{ }^{2}+z^{2}}}\right] \\
& =\frac{\mu_{0}}{4 \pi} \ln \left(\frac{p_{-}}{p_{+}}\right)^{2} I \hat{e}_{z}
\end{aligned}
$$

as is shown by $D$. Carson and P. Lorraine, "Intro. Electromag. Fields and Waves," W.H. Freeman and Co., S.F., Eqn. (5-70), p. 189.

For the configuration shown, we perform the integration over $y$ (from $-h$ to $+h)$

$$
\begin{aligned}
& A_{z} / J=\frac{\mu_{0}}{4 \pi} \int_{-h}^{h} d y \ln \left\{\frac{[X-(s+p)]^{2}+(y-Y)^{2}}{[X-(s-p)]^{2}+(y-Y)^{2}} \frac{[X+(s+p)]^{2}+(y-Y)^{2}}{[X+(s-p)]^{2}+(y-Y)^{2}}\right\}
\end{aligned}
$$

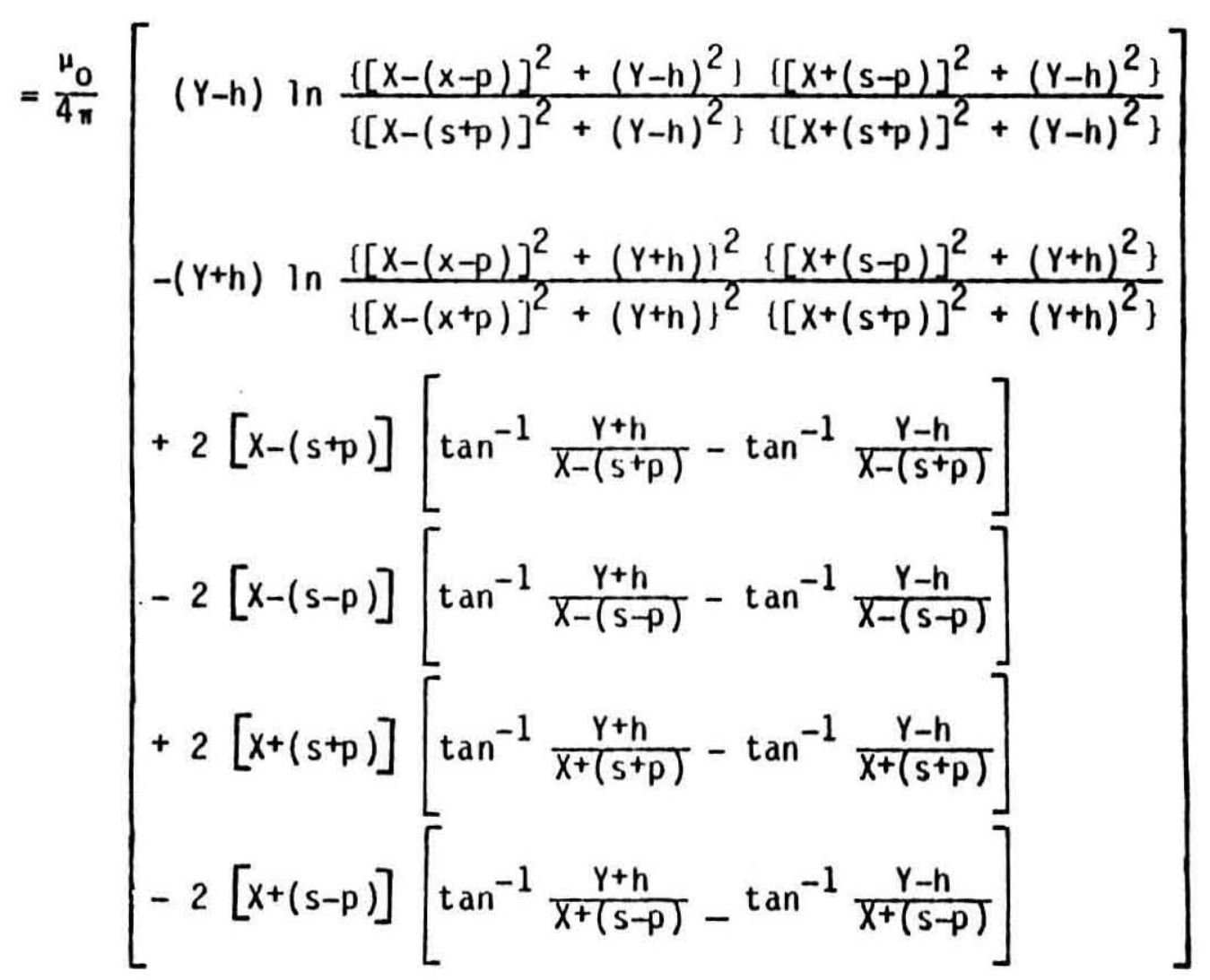

14 
With $\mu_{0} / 4 \pi=0.1$ this is programmed as AZCJ1 in Program FORPP.

For $\mathrm{p}$ and $\mathrm{h}$ small, the configuration might be approximated as two parallel 2-D strips of magnetic moment. We note that for an individual magnetic dipole of moment $|\vec{m}|=I$ - Area, $\vec{A}=\mu_{0} / 4 \pi\left(\vec{m} \times \vec{R} / R^{3}\right)$. Here, for one pair of current strips, we have a magnetic moment per unit length of 4Jph -- to be integrated over $z$ from $-\infty$ to $\infty$ to obtain:

For one pair of current strips,

$$
A_{z} / J \propto \frac{\mu_{0}}{\pi} p h \int_{-\infty}^{\infty} \frac{(x-s)}{\left[(x-s)^{2}+y^{2}+z^{2}\right]^{3 / 2}}=-\frac{2 \mu_{0}}{\pi} \text { ph } \frac{x-s}{(x-s)^{2}+y^{2}}
$$

and for the two pair

$$
A_{z} / J=-\frac{2 \mu_{0}}{\pi}\left[\frac{x-s}{(x-s)^{2}+y^{2}}-\frac{x+s}{(x+s)^{2}+y^{2}}\right] .
$$

With $2 \mu_{0} / \pi=8\left(\mu_{0} / 4 \pi\right)=0.8$, this is programmed (as a check) as AZCU2 in Program FORPP.

To solve the same problem on "POISSON" we take into account the finite nature of the mesh density, especially along the current sheets.

$$
\begin{aligned}
h & \equiv \text { current sheet height. } \\
N & \equiv \text { No. of nodes on the sheet. } \\
I_{0} & \equiv \text { current at each node. } \\
\delta & \equiv \text { nodal spacing. } \\
J & \equiv \text { current density. }
\end{aligned}
$$




$$
I_{0}=J \quad \delta ; \frac{h+\frac{1}{2} \delta}{N}=\delta \Rightarrow h=\delta\left(N-\frac{1}{2}\right)
$$

If we take:

$$
\begin{gathered}
I_{0}=5 ; \\
N=30 ; \\
J=N-1=29 \\
h=\frac{29.5}{29.0} \times 5=5.0862 \\
\delta=\frac{5}{29}
\end{gathered}
$$

we get

The analytical method solves for the vector potential $A / J_{0}$, and the relaxation method solves for $A_{r e}$. If both cases use the same geometry their vector potentials are related:

$$
\left(\frac{A}{J_{0}}\right)_{\text {analytical }}=\left(\frac{A}{J_{i}}\right)_{\text {relaxation }}
$$

$$
\mathrm{J}_{i_{\text {relax. }}}=\frac{A_{\text {relax }}}{(\text { ATJ })_{\text {analy. }}}
$$

or:

$$
\frac{{ }^{J_{\text {relax. }}}}{J_{0}}=\frac{\left(A / J_{0}\right)_{\text {analy. }}{ }^{\text {relax. }}}{J_{0}}
$$

If the two solutions are the same, $\mathrm{J}_{i \text { relax. }} / \mathrm{J}_{0}=1$.

The relaxation method however is affected by the boundary conditions which, if incorrect, introduces a certain error measured as the depature 
of $J_{i \text { relax }} / J_{0}$ from unity in Eq. (1). Table 1 lists results for several different $x, y$ locations, and boundary conditions. Figure 4 is a plot of these results. Figure 5 shows the flux lines along the boundary for the 3 types of b.c. used. In each case the use of the procedure suggested here appears to give results that distinctly are to be preferred. 


\begin{tabular}{|c|c|c|c|c|c|c|c|c|c|}
\hline \multirow[b]{2}{*}{$x(\mathrm{~cm})$} & \multirow[b]{2}{*}{$Y(\mathrm{~cm})$} & \multicolumn{2}{|c|}{ Analytical Solution } & \multicolumn{2}{|l|}{ Our B.C. } & \multicolumn{2}{|c|}{ Dirichlet B.C. } & \multicolumn{2}{|c|}{ Neumann B.C. } \\
\hline & & $\mathrm{A} / \mathrm{J}_{0}$ & $\frac{A-A(0,0)}{J_{0}}$ & $A_{\text {relax }}-A_{\text {relax }}^{(0,0)}$ & $\mathrm{J}_{\mathrm{i}} / \mathrm{J}_{0}$ & $A_{\text {relax }}-A_{\text {relax }}^{(0,0)}$ & $J_{i} / J_{0}$ & $A_{\text {relax }}-A_{\text {relax }}^{(0,0)}$ & $\mathrm{J}_{\mathrm{i}} / \mathrm{J}_{0}$ \\
\hline 0 & 0 & 2.431588 & 0 & 0 & - & 0 & - & 0 & - \\
\hline 1.0 & 0 & 2.455497 & .023909 & -0.74 & 1.068 & -0.80 & 1.15 & -0.6 & 0.864 \\
\hline 4.0 & 0 & 2.828064 & .396476 & -12.55 & 1.092 & -14.4 & 1.25 & -10.8 & 0.939 \\
\hline 8.0 & 0 & .1928442 & -2.238744 & +65.85 & 1.014 & +58.1 & 0.894 & +72.8 & 1.12 \\
\hline 12.8 & 0 & -1.074990 & -3.506578 & +104.45 & 1.028 & +82.65 & 0.812 & +123.4 & 1.21 \\
\hline 13.8 & 0 & -.9190218 & -3.350610 & +99.55 & 1.024 & +74.46 & 0.766 & +122.6 & 1.26 \\
\hline 0.213 & 13.998 & .6383113 & -1.793277 & +54.75 & 1.052 & +72.9 & 1.40 & +34.3 & 0.659 \\
\hline 4.494 & 12.198 & .6432313 & -1.788357 & +54.25 & 1.046 & +68.27 & 1.316 & +38.3 & 0.738 \\
\hline 4.601 & 10.110 & .8142468 & -1.617341 & +48.85 & 1.042 & +58.3 & 1.242 & +37.9 & 0.808 \\
\hline 9.007 & 9.701 & .2662448 & -2.165343 & +64.95 & 1.034 & +70.1 & 1.11 & +57.66 & 0.918 \\
\hline
\end{tabular}

Table 1 
effect of boundary conditions

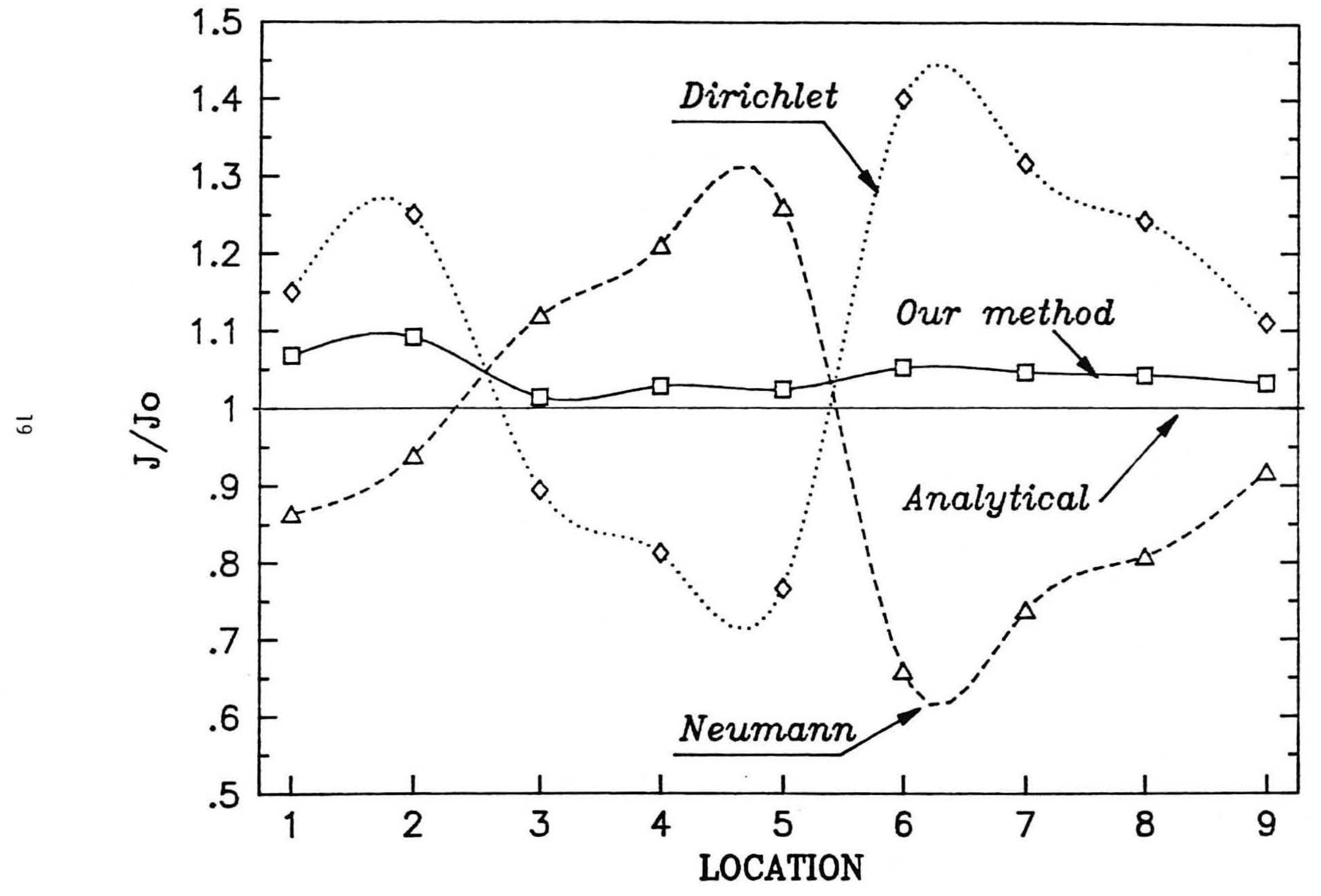

Fig. 4 

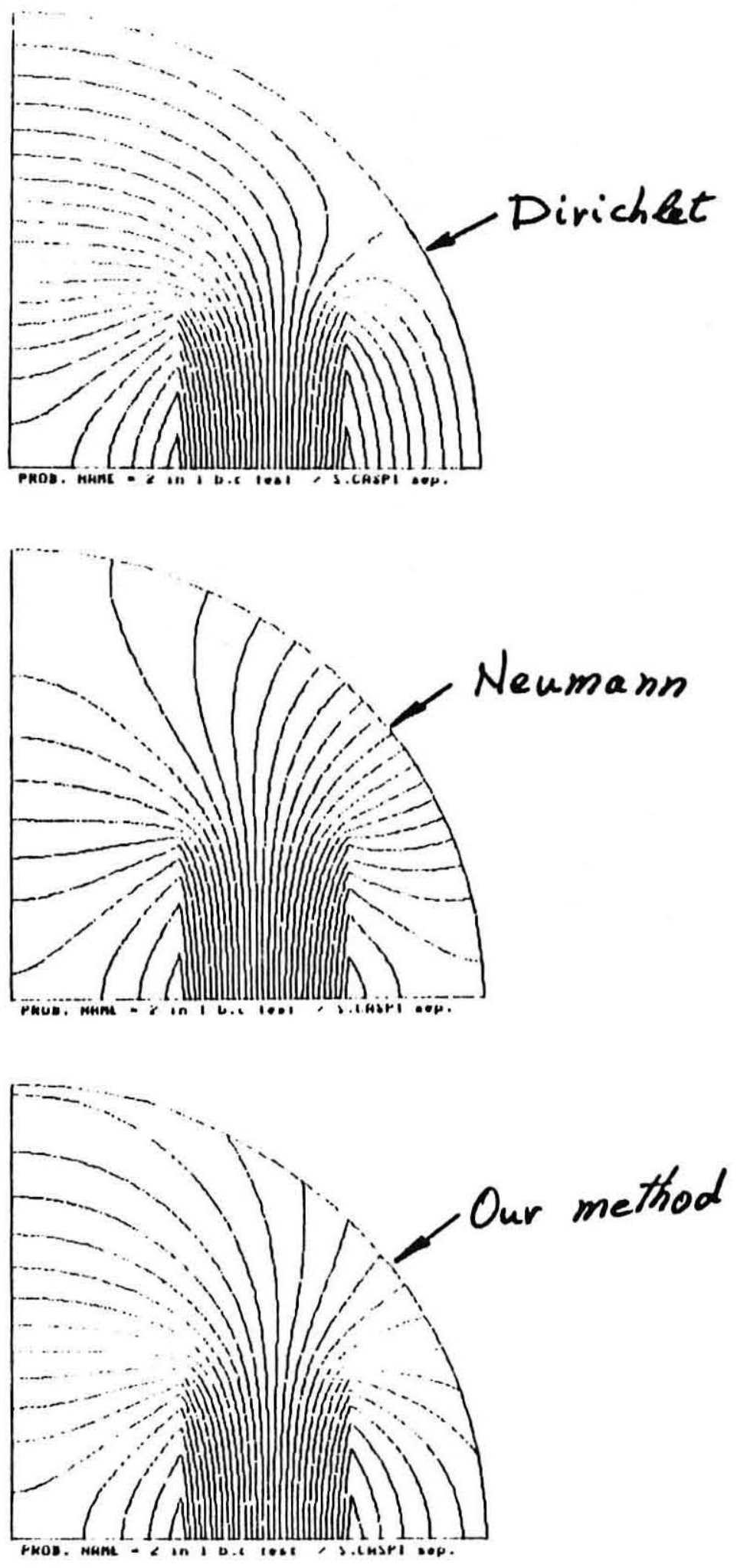

Fig. 5. Flux lines using the three different types of boundary conditions. 
Single Dipole Quadrupole and Sextupole Using Line Current

The analytical solution of the vector potential for these types of problems is outlined in LBID-847 (program "POTEN" on the HP 1000). The flux lines of the three problems solved by POISSON using our method is shown in Fig. 6. Table 2 compares the POISSON values with those solved analytically at some arbitrary locations.

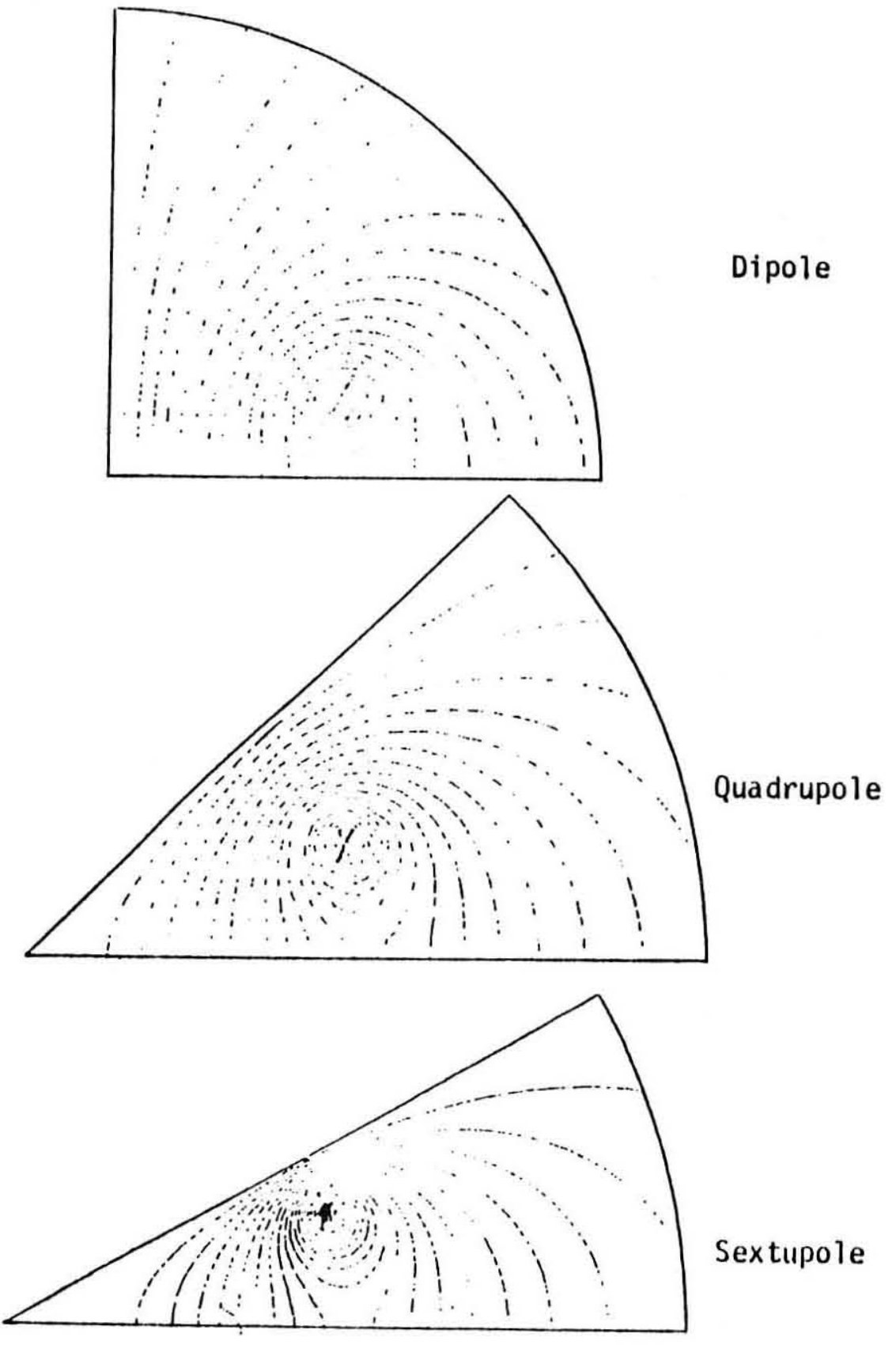

Fig. 6 


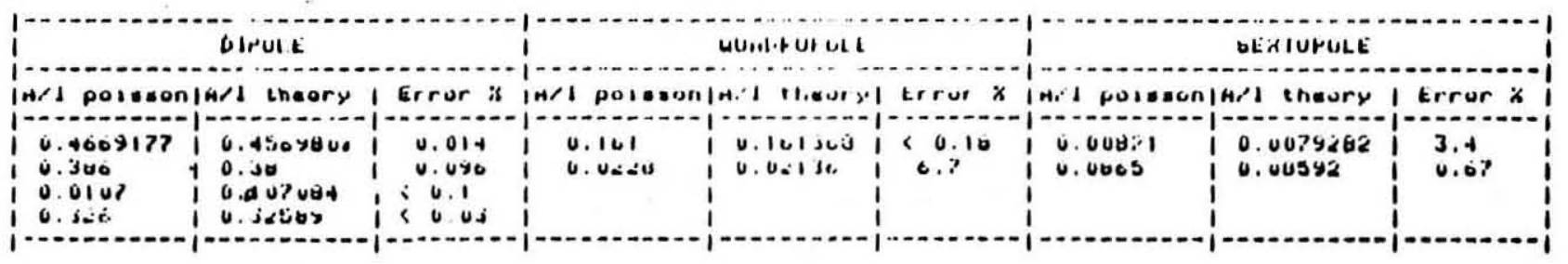

Table 2

\section{Erroneous Results}

In the course of studying a 2 in 1 magnet for the SSC we came across a case which clearly shows the improvement of using the new boundary method. In this case the two iron clampet dipoles of which only one is energized have been placed inside a thin iron cryostat. An imaginary circular boundary outside the cryostat confines the mesh size of the problem. When a Dirichlet boundary condition is imposed the solution result with flux lines circulating the iron cryostat as if a final net current flows through the dipole, Fig. 7a. In the case when the boundary condition has been replaced with a Neumann type, premature return of flux lines occur, Fig. 7b. Our results are shown in Fig. 7c. 

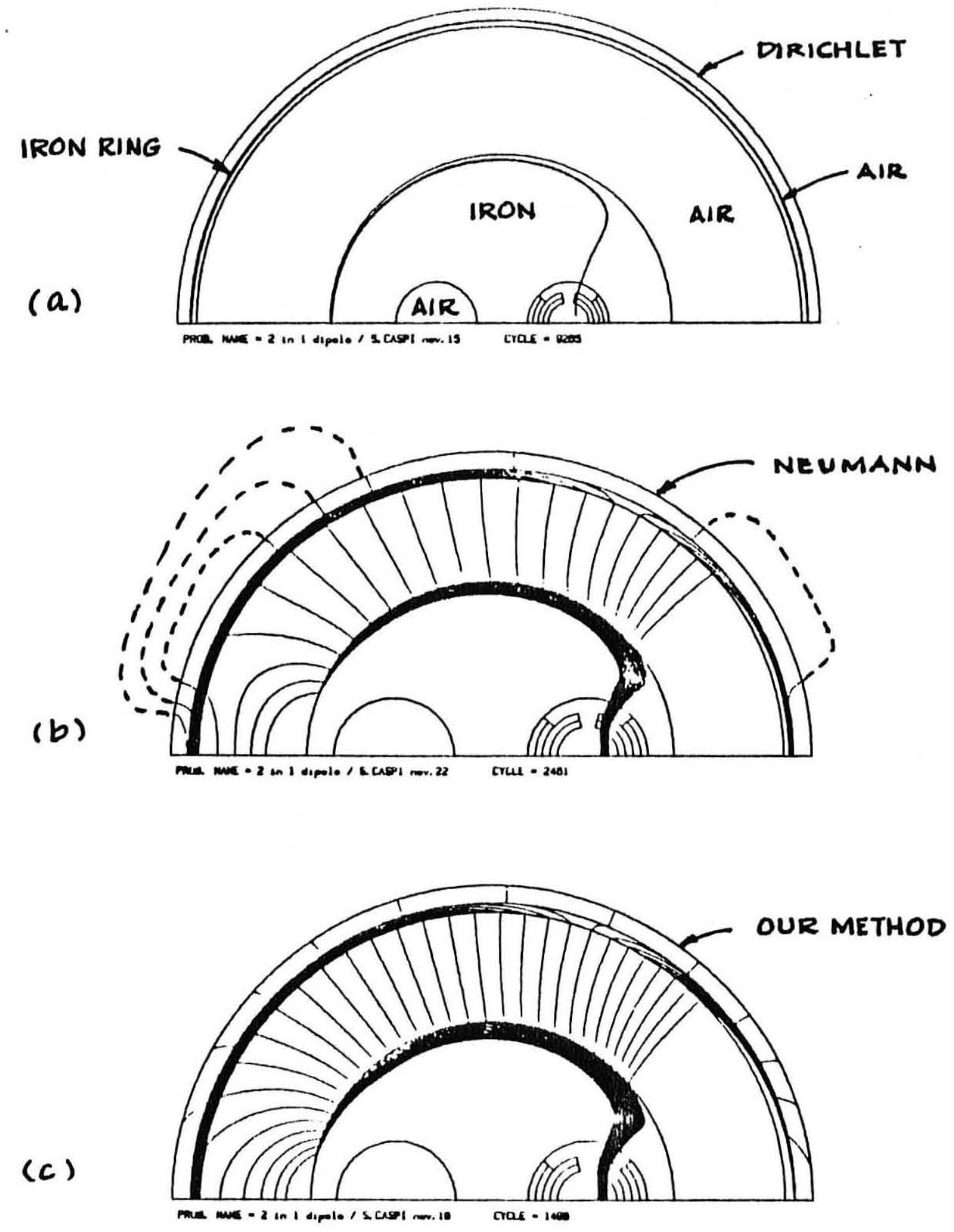

Fig. 7. A non-physical boundary condition can result in a non-physical solution. On top (case a) a Dirichlet condition results in flux lines circling the iron ring as if a net current flows through the dipole. In the center (case b) a Neumann condition results in flux lines which are prematurely returned to the iron ring. The correct flux lines are shown in the bottom (case c) using our method. It is however possible that when the boundary is farther removed from the iron ring, increasing the air and overall problem mesh size, both cases $a$ and $b$ can end up with reasonable flux 1 ines. 
How to Use the New Boundary Condition

This section describes how the user incorporates the "universe" boundary condition into a POISSON-type problem. (The method used is described in the analys is section under the Least Square Method.) The POISSON group originating from the VAX has been modified to our HP 1000 computer specifications. Experience has shown that any problem which converges using the standard boundary conditions (Dirichlet or Neumann) will converge when the universe boundary condition is used. Problems that converge with difficulty, or not at all may experience similar difficulties using the new boundary condition.

We follow a standard sequence:

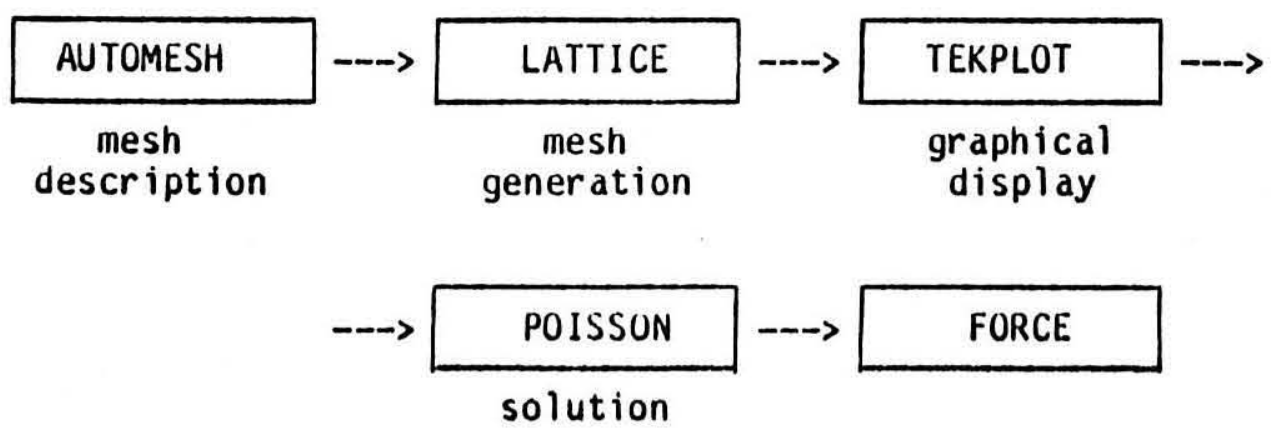

AUTOMESH

The user must add an additional region to the AUTOMESH input data. The new region may only be placed on the problem boundary. This region has the same general form as other regions in AUTOMESH input data: a 'REG' region description card, and a set of 'PO' geometry cards following. A description of these cards follows.

The region card must set the material code to 513. The current and current density variables must be set to 0.0 .

The geometry must conform to the following specifications: 
- The 'universe' is a shell consisting of two circular concentric arcs centered on the origin of the problem's coordinate system.

- The arcs must be far enough apart for at least one mesh triangle to fit between them.

- The shell is closed by any lines of symmetry in the problem (it is a ring if none exist).

- The shell must be placed outside all 'source' and 'iron' regions.

- The nodal spacing is not required to be equal.

Figure (1) demonstrates this geometry. No error checking of any kind is done for these specifications: (AUTOMESH may pass faulty "universe" regions without reporting any errors, but the problem will not make physical sense.) There is no restriction on how distant the shell may be from 'source' and 'iron' regions; the shell may also be thicker than one triangle. However, additional points increase the size (and execution time) of the relaxation process in POISSON. 
The following example of AUTOMESH input may help to clarify these points.

test b.c with new poisson 22 nov. 1983.

IREG NREG $=3$ MAT $=1 \quad X M A X=5.0 \quad$ YMAX $=5.0 N P O I N T=4$

SREG XMIN $=-3.0 E-6 \quad Y M I N=-3.0 \mathrm{e}-6$

\$REG $d x=0.160 \times$ regl $=0.5$ yregl $=2$.

\$REG yreg2 $=3.0$ xreg $2=2.5 \$$

\$PO $X=0.0 \quad Y=0.0 \$$

\$PO nt $=1 \times 0=0.0$ y0 $=0.0 \quad r=5.0$ thet $a=0.0$ \&

\$PO $n \mathrm{t}=2 \times 0=0.0$ y $0=0.0 \mathrm{r}=5.0$ thet $\mathrm{a}=90$. \$

\$po $x=0.0 \quad y=0.0 \&$

SREG MAT $=1$ cur $=-1000$. NPOINT $=5 \$$

Spo $x=1.0 y=0.0$ \&

Spo $x=2.0 y=0.0 \mathrm{~s}$

Spo $x=2.0 \quad y=1.0 \$$

\$po $x=1.0 y=1.0 \$$

\$po $x=1.0 \quad y=0.0 \&$

\$reg mat $=513$ cur $=0.0$ den $=0.0$ npoint $=5 \&$

\&po $n t=1 \times 0=0$ y0=0.0 $r=4.60$ thet $a=0.0$ \&

Spo $n t=1 \times 0=0$ y $0=0.0 r=5.0$ thet $a=0.0$ \&

\$po $n t=2 \times 0=0.0$ y0=0.0 $r=5.0$ thet a 90.0 \&

\$po $n t=1 \times 0=0.0$ y0 $=0.0 r=4.60$ thet $a=90.0 \&$

\&po $n t=2 \times 0=0.0$ y0=0.0 $r=4.60$ the $t a=0.0 \&$
: Title card

: First REG card

: Multiple zoning

: Problem geometry

: Conductor region

: Universe region

\section{!}




\section{LATTICE AND TEKPLOT}

The "universe" region is just another air region to LATTICE and TEKPLOT. No special user intervention is required. TEKPLOT cannot distingush the "universe" region from adjacent air regions. They will appear to be one and the same.

\section{POISSON}

Five new problem constants (the so-called CONs) have been added to POISSON.

$\operatorname{CON}(126)$ is the key to the use of the "universe" boundary condition in POISSON. This CON determines the symmetry type of the "universe" region (1t is the basis for the $\alpha$ 's used in the Analysis section). It is unrelated to the problem symmetry type $\operatorname{CONs}(\operatorname{CON}(46)$ and $\operatorname{CON}(19))$. If $\operatorname{CON}(126)$ is less than or equal to zero, the universe boundary condition is not used, coefficients are not calculated, and no adjustment of the outer arc potentials will take place. This means that a problem may be run with the standard (Dirichlet or Neumann) boundary conditions initially. (These are determined by CONs 21-25). Once $\operatorname{CON}(126)$ is set and the $E$ matrix is calculated, it is not possible to return the outer boundary of the shell to standard boundary conditions. If $\operatorname{CON}(126)$ is reset to zero the potentials on the outer arc of the shell are frozen.

$$
\text { Default is } \operatorname{CON}(126)=0 \text {. }
$$


Supported Symmetry Types

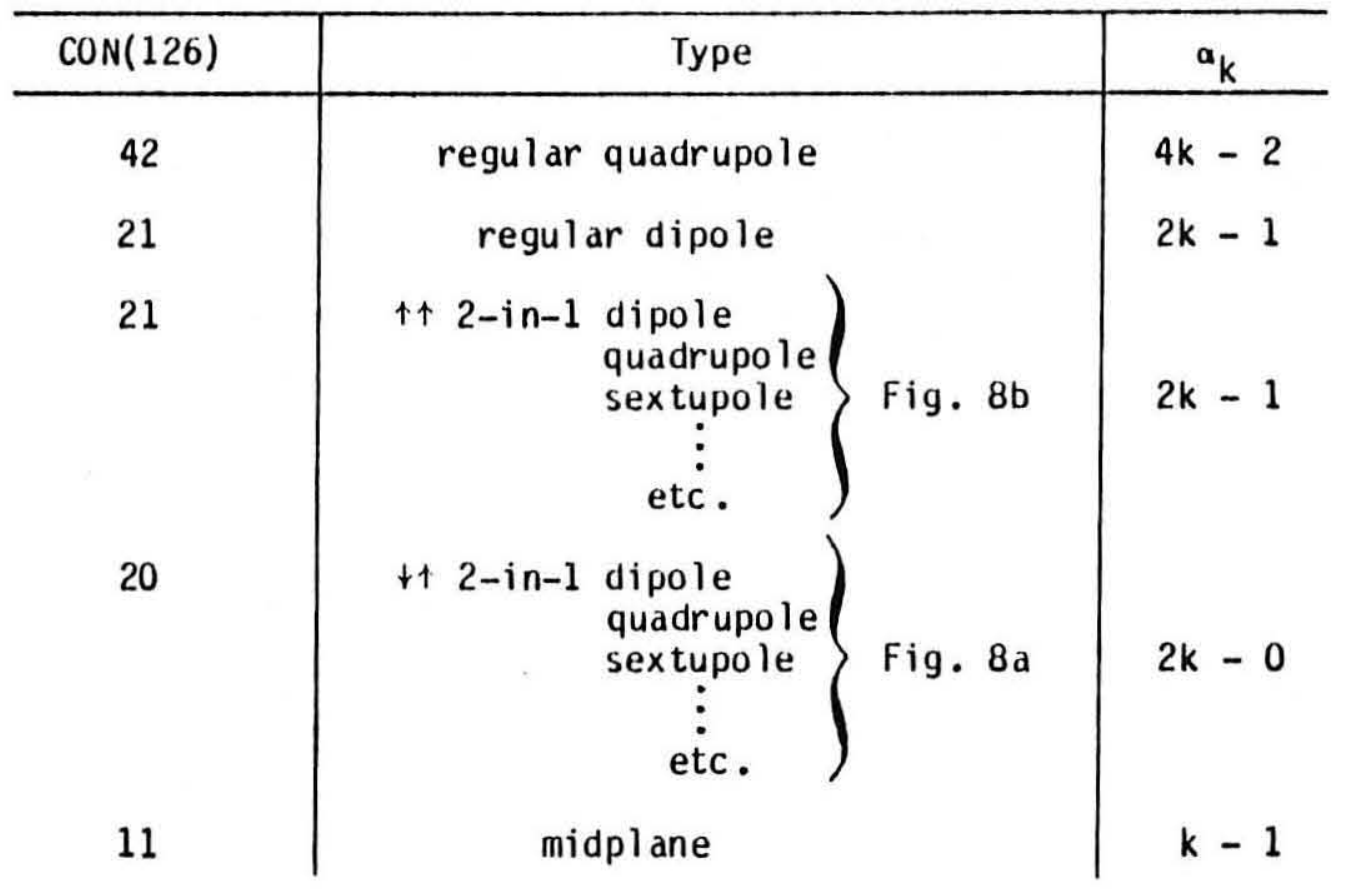

$\operatorname{CON}(129)$ determines how often to adjust the potentials on the outer arc. The adjustment is done every $(\operatorname{CON}(129))$ relaxation sweeps (cycles). If $\operatorname{CON}(129)$ is less than or equal to zero, the potentials on the outer arc are frozen -- no adjustment takes place. Default is $\operatorname{CON}(129)=0$.

Experience has shown that potentials on the outer arc stabilize quickly. while the rest of the problem may have many more cycles to go before satisfying the convergence criteria. Execution time of a large problem may be reduced by performing the adjustment less often.

The following example of POISSON input indy help to clarify these points. 


$\begin{array}{ll}0 \text { DUMP } & : \text { Start from LATTICE run } \\ \star 60 & : \text { Real iron } \\ \star 751.89 & : \text { Don't optimize relaxation factor... } \\ \star 462 & : \text {...this is sometimes helpful } \\ \star 12611 & : \text { Problem symmetry (midplane) } \\ \star 12910 & : \text { Adjust every } 10 \text { cycles } \\ -1 \text { DUMP } & : \text { End }\end{array}$

$\operatorname{CON}(127)$ and $\operatorname{CON}(128)$ contain the dimensions of the 'working matrix' $E$. This matrix is calculated, then saved at the end of that dump. A problem. requiring multiple POISSON runs will not need to recalculate the 'working matrix'. These cons should not be altered by the user.

$\operatorname{CON}(130)$ is reserved for future use.

FORCE

Like LATTICE and TEKPLOT, FORCE cannot distinguish the "universe" region from an air region. No user adjustments are necessary.

Other POISSON Prograins

These features have not been incorporated into any other programs in the POISSON group. 

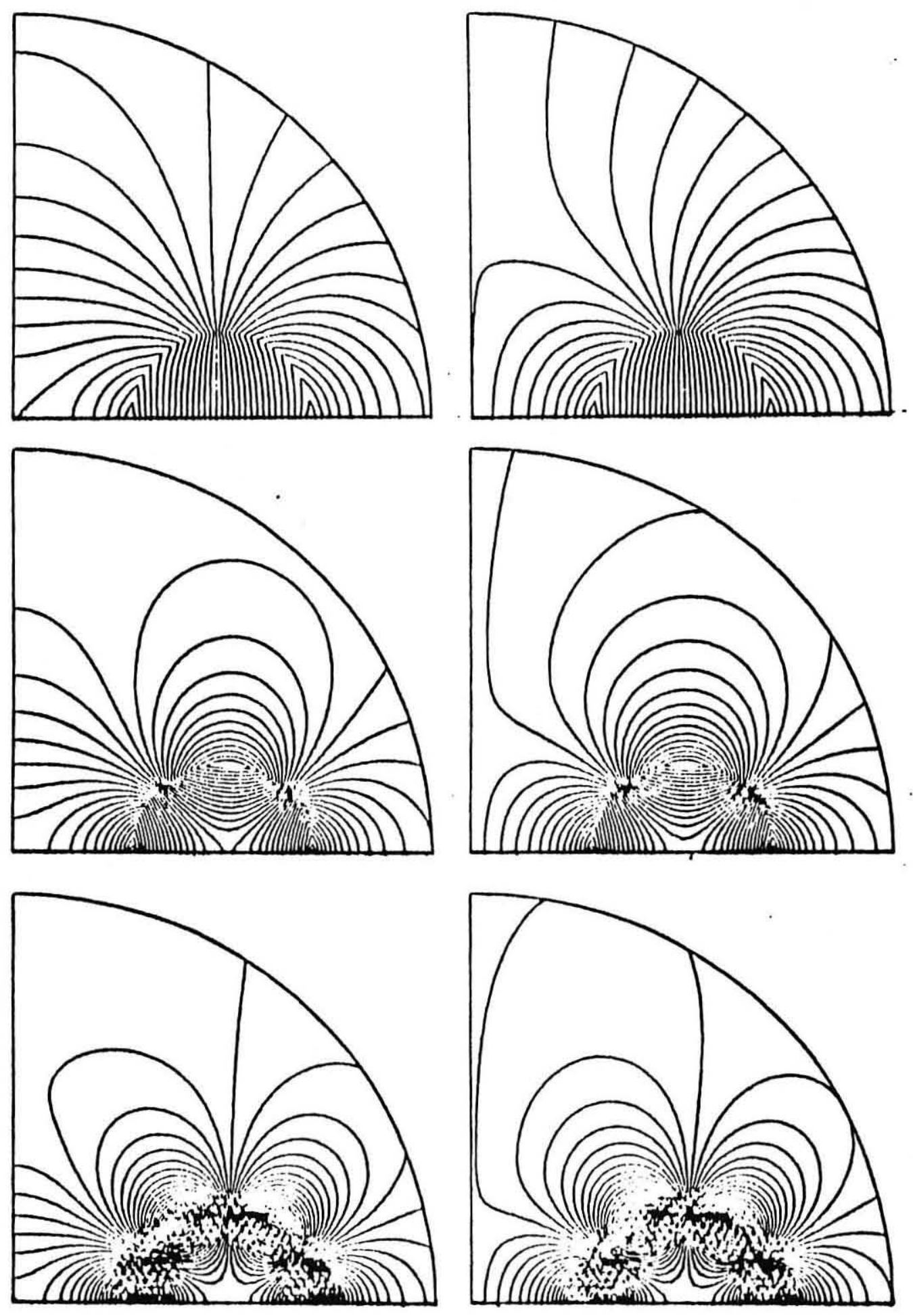

a $(\downarrow \uparrow)$

b $(\uparrow \uparrow)$

Fig. 8 Two in one, dipole, quadrupole and sextupole, emphasizing the type of boundary conditions. 